Article

\title{
Approximate Mei Symmetries and Invariants of the Hamiltonian
}

\author{
Umara Kausar $^{\dagger}$ and Tooba Feroze $*,+(D)$ \\ School of Natural Sciences, National University of Sciences and Technology, Sector H-12, \\ Islamabad 44000, Pakistan; ukausar.phdmath19sns@student.nust.edu.pk \\ * Correspondence: tferoze@sns.nust.edu.pk \\ † These authors contributed equally to this work.
}

Citation: Kausar, U.; Feroze, T.

Approximate Mei Symmetries and

Invariants of the Hamiltonian.

Mathematics 2021, 9, 2910. https:/ /

doi.org/10.3390/math9222910

Academic Editors: Andrei

Dmitrievich Polyanin and

Alexander V. Aksenov

Received: 16 September 2021

Accepted: 21 October 2021

Published: 15 November 2021

Publisher's Note: MDPI stays neutral with regard to jurisdictional claims in published maps and institutional affiliations.

\begin{abstract}
It is known that corresponding to each Noether symmetry there is a conserved quantity. Another class of symmetries that corresponds to conserved quantities is the class of Mei symmetries. However, the two sets of symmetries may give different conserved quantities. In this paper, a procedure of finding approximate Mei symmetries and invariants of the perturbed/approximate Hamiltonian is presented that can be used in different fields of study where approximate Hamiltonians are under consideration. The results are presented in the form of theorems along with their proofs. A simple example of mechanics is considered to elaborate the method of finding these symmetries and the related Mei invariants. At the end, a comparison of approximate Mei symmetries and approximate Noether symmetries is also given. The comparison shows that there is only one common symmetry in both sets of symmetries. Hence, rest of the symmetries in the two sets correspond to two different sets of conserved quantities.
\end{abstract}

Keywords: approximate Noether symmetries; conservation laws; Hamiltonian

\section{Introduction}

Differential equations involving small parameters, called the perturbed term, often appear as mathematical models of real world problems. The parameter, generally, corresponds to some error or correction. Various methods have been developed to solve equations involving perturbed/approximate term e.g., the homotopy perturbation method, Adomain decomposition method, inverse scattering transformation method and approximate symmetries method. Baikov et al. [1] were the first to study the approximate groups of the perturbed differential equations and developed a theory based on approximate groups. Furthermore, the approximate Lie theorem provides a mechanism to calculate the approximate symmetries of perturbed differential equations. Gazizov [2] described some properties of approximate symmetries and developed an algorithm to calculate the approximate invariants.

Feroze and Kara [3] used the approximate symmetry generators and invariants of the perturbed ordinary differential equations (ODEs) to construct the Lagrangians. After that, Johnpillai and Kara [4] utilized the same approach to construct approximate Lagrangians for perturbed partial differential equations (PDEs). In [5], approximate symmetries and conserved quantities of a system of differential equations are discussed. In [6], approximate symmetries are calculated for the perturbed $\mathrm{KdV}$ equation and a one-dimensional subalgebra of an optimal system is obtained. Camci [7] presents third order approximate Noether symmetries for Bardeen spacetime and has formulated some new approximate Noether gauge symmetry relations for perturbed Lagrangians.

Feng-Xiang [8], used an infinitesimal transformation of groups to establish invariance of equations of motion. Dynamical functions such as Lagrangian, Hamiltonian etc., are replaced with transformed dynamical functions. This method, which preserves the form of equations of motion, is called form invariance, also known as Mei symmetries. In the 
context of infinitesimal transformation of a group, form invariance of Appell equations is investigated in [9]. The Lagrangian of the Appell equations is used to compute the Noether symmetries. The Noether symmetries are then compared to form invariance to obtain the different conserved quantities. Shu-Yong and Feng-Xiang in [10] investigated form invariance and Lie symmetries in a non-holonomic system. Structure equations and form invariance, which are similar to Lie symmetries, are deduced in this article. Jian-Hui [11], presents the Mei symmetries of a rotational relativistic mass variable system, with an emphasis on the relationship between the Lie and Mei symmetries. On a time scale, the Mei symmetries of the Lagrangian and Birkhoffian systems are presented in [12,13] and are thoroughly discussed and compared with the Noether symmetries. The Hamiltonian canonical equations are considered as a special case in the construction of the Mei symmetries of the Birkhoffian system.

Jia et al. [14] introduced some definitions and developed criterion to calculate the special Mei symmetries of Appell equations for weakly nonholonomic system and their relative approximate Mei invariants. The Appell equations are specifically established for nonholonomic system for these symmetries. The Lie symmetry and approximate Hojman conserved quantity of Appell equations are investigated for a weakly nonholonomic system by Han et al. [15]. Furthermore, under the infinitesimal transformation of group in which time is invariable, the Lie symmetries are found for weakly nonholonomic systems and their first degree approximate holonomic systems.

In this paper, we focus on the formulation of approximate Mei symmetries and invariants. The method is then used to find the exact and approximate Mei symmetries that arise from the Hamiltonian of the linear equation of motion of the damped harmonic oscillator (DHO). This Hamiltonian is calculated from the Lagrangian of the DHO equation which is given in [16]. The Legendre transformations are used to convert the Lagrangian system to the Hamiltonian system. The outline of paper is as follows. The determining equations of exact and approximate Mei symmetries and invariants are given in Theorems 1 and 2 in Section 1. Section 2 consists of the approximate Mei symmetries of DHO. In Section3, an example is given to elaborate the method. The paper is concluded in Section 4.

\section{Mei Symmetries of Approximate/Perturbed Hamiltonian}

The unperturbed Hamiltonian system has been discussed in terms of the Mei symmetries and their related first integrals in [17]. Here we present approximate Mei symmetries and invariants corresponding to perturbed Hamiltonian in the following Theorems 1 and 2.

Theorem 1. Let $\mathbf{Z}=\mathbf{Z}_{0}+\epsilon \mathbf{Z}_{1}$ be an approximate symmetry generator and $H=H_{0}+\epsilon H_{1}$ be the first order approximate Hamiltonian, where $\mathbf{Z}_{0}=\alpha_{0} \frac{\partial}{\partial t}+\beta_{0}^{a} \frac{\partial}{\partial x^{a}}$ and $\mathbf{Z}_{1}=\alpha_{1} \frac{\partial}{\partial t}+\beta_{1}^{a} \frac{\partial}{\partial x^{a}}$, then

$$
\begin{array}{cc}
E_{a}\left(\boldsymbol{Z}_{0}^{[1]} H_{0}\right)=0, & (a=1,2, \ldots, n) . \\
E_{a}\left(Z_{0}^{[1]} H_{1}+Z_{1}^{[1]} H_{0}\right)=0, \quad & (a=1,2, \ldots, n) .
\end{array}
$$

Here $E_{a}$ is called the Euler operator defined as

$$
E_{a}=\frac{d}{d t}\left(\frac{\partial}{\partial \dot{x}^{a}}\right)-\frac{\partial}{\partial x^{a}} \quad(a=1,2, \ldots, n) .
$$

Proof of Theorem 1. To prove the above relations, Equations (1) and (2), apply the first order prolongation of $\mathbf{Z}$, i.e., $\mathbf{Z}^{[1]}=\mathbf{Z}_{0}^{[1]}+\epsilon \mathbf{Z}_{1}^{[1]}$ on $H=H_{0}+\epsilon H_{1}$ to have

$$
\mathbf{Z}^{[1]} H=\left(\mathbf{Z}_{0}^{[1]}+\epsilon \mathbf{Z}_{1}^{[1]}\right)\left(H_{0}+\epsilon H_{1}\right)
$$

Neglecting the higher order terms in $\epsilon$ yields

$$
\mathbf{Z}^{[1]} H=\left(\mathbf{Z}_{0}^{[1]} H_{0}\right)+\epsilon\left(\mathbf{Z}_{0}^{[1]} H_{1}+\mathbf{Z}_{1}^{[1]} H_{0}\right) .
$$


Applying operator $E_{a}$, given in Equation (3), on Equation (5) and requiring the invariance $E_{a} Z^{[1]} H=0$, we have

$$
E_{a}\left(\mathbf{Z}_{0}^{[1]} H_{0}\right)+\epsilon E_{a}\left(\mathbf{Z}_{0}^{[1]} H_{1}+\mathbf{Z}_{1}^{[1]} H_{0}\right)=0
$$

Comparing the coefficients of different powers of $\epsilon$ leads to Equations (1) and (2). This completes the proof.

Theorem 2. Let $H=H_{0}+\epsilon H_{1}$ be the first order approximate Hamiltonian. If the symmetry generator $\mathbf{Z}=\alpha \frac{\partial}{\partial t}+\beta^{a} \frac{\partial}{\partial x^{a}}$ satisfies Theorem 1, then the first integrals (invariants) have the form given below

$$
\begin{array}{r}
I_{0}=\alpha_{0}\left(\mathbf{Z}_{0}^{[1]} H_{0}\right)+\left(\beta_{0}^{a}-\dot{x}^{a} \alpha_{0}\right) \frac{\partial\left(\mathbf{Z}_{0}^{[1]} H_{0}\right)}{\partial \dot{x}^{a}}, \\
I_{1}=\alpha_{0}\left(Z_{0}^{[1]} H_{1}+Z_{1}^{[1]} H_{0}\right)+\alpha_{1}\left(Z_{0}^{[1]} H_{0}\right)+\left(\beta_{1}^{a}-\dot{x}^{a} \alpha_{1}\right) \frac{\partial\left(\mathbf{Z}_{0}^{[1]} H_{0}\right)}{\partial \dot{x}^{a}} \\
+\left(\beta_{0}^{a}-\dot{x}^{a} \alpha_{0}\right) \frac{\partial\left(Z_{0}^{[1]} H_{1}+Z_{1}^{[1]} H_{0}\right)}{\partial \dot{x}^{a}} .
\end{array}
$$

Proof of Theorem 2. To establish the aforementioned statements, we consider the Noether identity $\alpha H+\left(\beta^{a}-\dot{x}^{a} \alpha\right) \frac{\partial H}{\partial \dot{x}^{a}}$, in terms of the Hamiltonian, which is obtained from the Lagrangian by using the Legendre transformations defined as

$$
H_{0}+\epsilon H_{1}=p_{k} \dot{q}^{k}-\left(L_{0}+\epsilon L_{1}\right)
$$

Now, applying differential operator $\mathbf{Z}^{[1]}$ on the Noether identity, we obtain

$$
I=\alpha\left(\mathbf{Z}^{[1]} H\right)+\left(\beta^{a}-\dot{x}^{a} \alpha\right) \frac{\partial\left(\mathbf{Z}^{[1]} H\right)}{\partial \dot{x}^{a}} .
$$

In the preceding Equation (10), taking the perturbed invariant up to the first order of $\epsilon$, i.e., $I_{0}+\epsilon I_{1}, \mathbf{Z}=\mathbf{Z}_{0}+\epsilon \mathbf{Z}_{1}$, and $H=H_{0}+\epsilon H_{1}$ we have

$$
\begin{aligned}
I_{0}+\epsilon I_{1}=\left(\alpha_{0}+\epsilon \alpha_{1}\right)\left[\left(\mathbf{Z}_{0}^{[1]}+\epsilon \mathbf{Z}_{1}^{[1]}\right)\left(H_{0}+\epsilon H_{1}\right)\right]+ & {\left[\left(\beta_{0}^{a}+\epsilon \beta_{1}^{a}\right)-\dot{x}^{a}\left(\alpha_{0}+\epsilon \alpha_{1}\right)\right] } \\
& \frac{\partial\left(\mathbf{Z}_{0}^{[1]}+\epsilon \mathbf{Z}_{1}^{[1]}\right)}{\partial \dot{x}^{a}}\left(H_{0}+\epsilon H_{1}\right) .
\end{aligned}
$$

Rearranging the above expression as

$$
\begin{aligned}
I_{0}+\epsilon I_{1}=\alpha_{0}\left(\mathbf{Z}_{0}^{[1]} H_{0}\right)+ & \epsilon\left[\alpha_{0}\left(\mathbf{Z}_{0}^{[1]} H_{1}+\mathbf{Z}_{1}^{[1]} H_{0}\right)+\alpha_{1}\left(\mathbf{Z}_{0}^{[1]} H_{0}\right)\right]+\left(\beta_{0}^{a}-\dot{x}^{a} \alpha_{0}\right) \frac{\partial\left(\mathbf{Z}_{0}^{[1]} H_{0}\right)}{\partial \dot{x}^{a}} \\
& +\epsilon\left[\left(\beta_{1}^{a}-\dot{x}^{a} \alpha_{1}\right) \frac{\partial\left(\mathbf{Z}_{0}^{[1]} H_{0}\right)}{\partial \dot{x}^{a}}+\left(\beta_{0}^{a}-\dot{x}^{a} \alpha_{0}\right) \frac{\partial\left(\mathbf{Z}_{0}^{[1]} H_{1}+\mathbf{Z}_{1}^{[1]} H_{0}\right)}{\partial \dot{x}^{a}}\right] .
\end{aligned}
$$

Separating powers of $\epsilon$ up to first order, we obtain the expressions given in Equations (7) and (8). This completes the proof.

\section{Mei Symmetries of the Approximate Hamiltonian of DHO}

The linear equation of motion of DHO is taken as an example. Using Legendre transformations described in Equation (9), the Hamiltonian of DHO is

$$
H\left(t, y, y^{\prime}\right)=\frac{1}{2}\left(y^{\prime 2}+y^{2}\right)+\epsilon t\left(y^{\prime 2}+y^{2}\right) .
$$


Now, writing $H$ by separating the powers of $\epsilon$, we obtain

$$
\begin{aligned}
& H_{0}=\frac{1}{2}\left(y^{\prime 2}+y^{2}\right), \\
& H_{1}=t\left(y^{\prime 2}+y^{2}\right) .
\end{aligned}
$$

Applying $\mathbf{Z}_{0}^{[1]}$ on Equation (14a), we obtain

$$
\mathbf{z}_{0}^{[1]} H_{0}=y^{\prime} \beta_{0, t}+y^{\prime 2} \beta_{0, y}-y^{\prime 2} \alpha_{0, t}-y^{\prime 3} \alpha_{0, y}+y \beta_{0} .
$$

Now Equation (1) ( $a=1)$, for the above Equation (15), gives

$$
E_{1}\left(\mathbf{Z}_{0}^{[1]} H_{0}\right)=0 .
$$

Alternatively,

$$
\frac{d}{d t}\left(\frac{\partial \mathbf{Z}_{0}^{[1]} H_{0}}{\partial y^{\prime}}\right)-\left(\frac{\partial \mathbf{Z}_{0}^{[1]} H_{0}}{\partial y}\right)=0 .
$$

The above Equation (17) yields the following expression

$$
\begin{array}{r}
\beta_{0, t t}+2 y^{\prime} \beta_{0, t y}+2 y^{\prime \prime} \beta_{0, y}-2 y^{\prime \prime} \alpha_{0, t}+y^{\prime 2} \beta_{0, y y}-2 y^{\prime} \alpha_{0, t t} \\
-4 y^{\prime 2} \alpha_{0, t y}-6 y^{\prime \prime} y^{\prime} \alpha_{0, y}-2 y^{\prime 3} \alpha_{0, y y}-\beta_{0}-y \beta_{0, y}=0 .
\end{array}
$$

Using $y^{\prime \prime}-y=0$ in Equation (18), we have

$$
\begin{gathered}
\beta_{0, t t}+2 y^{\prime} \beta_{0, t y}+2 y \beta_{0, y}-2 y \alpha_{0, t}+y^{\prime 2} \beta_{0, y y}-2 y^{\prime} \alpha_{0, t t} \\
-4 y^{\prime 2} \alpha_{0, t y}-6 y y^{\prime} \alpha_{0, y}-2 y^{\prime 3} \alpha_{0, y y}-\beta_{0}-y \beta_{0, y}=0 .
\end{gathered}
$$

The coefficients of different powers of $y^{\prime}$ yield the following system of PDEs

$$
\begin{aligned}
\beta_{0, t t}-\beta_{0}+y \beta_{0, y}-2 y \alpha_{0, t} & =0, \\
\beta_{0, t y}-\alpha_{0, t t}-3 y \alpha_{0, y} & =0, \\
\beta_{0, y y}-4 \alpha_{0, t y} & =0, \\
\alpha_{0, y y} & =0 .
\end{aligned}
$$

Equations (23) and (22), respectively, imply

$$
\begin{array}{r}
\alpha_{0}(t, y)=y f(t)+g(t), \\
\beta_{0}(t, y)=\frac{y^{2}}{2} f_{,}+y \delta(t)+\gamma(t) .
\end{array}
$$

Using Equations (24) and (25) in Equations (20) and (21), we obtain the following system

$$
\begin{aligned}
f_{, t t t} & =0, & \delta_{, t t}-2 g_{, t} & =0, \\
\gamma_{, t t}-\gamma(t) & =0, & \delta_{, t}-g_{, t t} & =0 .
\end{aligned}
$$

Solving the above system given in Equation (26) and substituting the solution in Equations (24) and (25) yields

$$
\begin{aligned}
& \alpha_{0}=C_{1}+e^{\sqrt{2} t} C_{2}+e^{-\sqrt{2} t} C_{3}, \\
& \beta_{0}=y \sqrt{2} e^{\sqrt{2} t} C_{2}-y \sqrt{2} e^{-\sqrt{2} t} C_{3}+e^{-t} C_{4}+e^{t} C_{5}+y C_{6} .
\end{aligned}
$$


Equation (27) provides the following list of symmetries viz.,

$$
\begin{aligned}
& \mathbf{Z}_{0}^{1}=\frac{\partial}{\partial t^{\prime}} \\
& \mathbf{Z}_{0}^{2}=e^{\sqrt{2} t} \frac{\partial}{\partial t}+y \sqrt{2} e^{\sqrt{2} t} \frac{\partial}{\partial y^{\prime}} \\
& \mathbf{Z}_{0}^{3}=e^{-\sqrt{2} t} \frac{\partial}{\partial t}-y \sqrt{2} e^{-\sqrt{2} t} \frac{\partial}{\partial y^{\prime}} \\
& \mathbf{Z}_{0}^{4}=e^{-t} \frac{\partial}{\partial y^{\prime}} \\
& \mathbf{Z}_{0}^{5}=e^{t} \frac{\partial}{\partial y}, \\
& \mathbf{Z}_{0}^{6}=y \frac{\partial}{\partial y} .
\end{aligned}
$$

Approximate Mei symmetries are calculated using the above exact symmetries given by Equations (28)-(33). For this, we consider $\mathbf{Z}_{0}^{3}$, to illustrate the method, where $\mathbf{Z}_{0}^{[1]} H_{1}+$ $\mathbf{Z}_{1}^{[1]} H_{0}$ is expressed as

$$
\begin{array}{r}
\mathbf{Z}_{0}^{[1]} H_{1}+\mathbf{Z}_{1}^{[1]} H_{0}=y^{\prime} \beta_{0, t}+y^{\prime 2} \beta_{0, y}-y^{\prime 2} \alpha_{0, t}-y^{\prime 3} \alpha_{0, y}+y \beta_{0}+y^{\prime 2} e^{-\sqrt{2} t} \\
+y^{2} e^{-\sqrt{2} t}+2 \sqrt{2} t y^{2} e^{-\sqrt{2} t}+4 t y y^{\prime} e^{-\sqrt{2} t} .
\end{array}
$$

Now using Equation (34) in Equation (2), we have

$$
\frac{d}{d t}\left(\frac{\partial\left(\mathbf{Z}_{0}^{[1]} H_{1}+\mathbf{Z}_{1}^{[1]} H_{0}\right)}{\partial y^{\prime}}\right)-\left(\frac{\partial\left(\mathbf{Z}_{0}^{[1]} H_{1}+\mathbf{Z}_{1}^{[1]} H_{0}\right)}{\partial y}\right)=0,
$$

or

$$
\begin{array}{r}
\beta_{1, t t}+2 y^{\prime} \beta_{1, t y}+2 y^{\prime \prime} \beta_{1, y}-2 y^{\prime \prime} \alpha_{1, t}+y^{\prime 2} \beta_{1, y y}-2 y^{\prime} \alpha_{1, t t}-4 y^{\prime 2} \alpha_{1, t y}-6 y^{\prime \prime} y^{\prime} \alpha_{1, y} \\
-2 y^{\prime 3} \alpha_{1, y y}-\beta_{1}-y \beta_{1, y}-2 \sqrt{2} y^{\prime} e^{-\sqrt{2} t}+4 y e^{-\sqrt{2} t}=0 .
\end{array}
$$

Using standard procedure of comparing the coefficients of different powers of $y^{\prime}$, the obtained system is

$$
\begin{aligned}
\beta_{1, t t}-\beta_{1}+y \beta_{1, y}-2 y \alpha_{1, t}+4 y e^{-\sqrt{2} t} & =0, \\
\beta_{1, t y}-\alpha_{1, t t}-3 y \alpha_{1, y}-\sqrt{2} e^{-\sqrt{2} t} & =0, \\
\beta_{1, y y}-4 \alpha_{1, t y} & =0, \\
\alpha_{1, y y} & =0 .
\end{aligned}
$$

Equations (39) and (40), imply

$$
\begin{array}{r}
\alpha_{1}(t, y)=y f(t)+g(t), \\
\beta_{1}(t, y)=\frac{y^{2}}{2} f_{, t}+y \delta(t)+\gamma(t) .
\end{array}
$$

Using Equations (41) and (42) in Equations (37) and (38), we obtain the following system

$$
\begin{aligned}
f_{, t t t} & =0, & \delta_{, t t}-2 g_{, t}+4 e^{-\sqrt{2} t} & =0, \\
\gamma, t t-\gamma(t) & =0, & \delta_{, t}-g_{, t t}-\sqrt{2} e^{-\sqrt{2} t} & =0 .
\end{aligned}
$$


Solving the above system given in Equation (43) and substituting the solution into Equations (41) and (42) yields

$$
\begin{aligned}
& \alpha_{1}=C_{1}+e^{\sqrt{2} t} C_{2}+e^{-\sqrt{2} t} C_{3}-\frac{1}{2} t e^{-\sqrt{2} t} \\
& \beta_{1}=y \sqrt{2} e^{\sqrt{2} t} C_{2}-y \sqrt{2} e^{-\sqrt{2} t} C_{3}+e^{-t} C_{4}+e^{t} C_{5}+y C_{6}-\frac{3}{2} y e^{-\sqrt{2} t}+\frac{1}{\sqrt{2}} y t e^{-\sqrt{2} t} .
\end{aligned}
$$

Now assigning value of any constant equal to one, say $C_{3}=1$, and the remaining constants equal to zero, we obtain the generator $\mathbf{Z}_{0}^{3}$ given in Equation (30). Then, $\mathbf{Z}_{1}^{3}$ can be written as

$$
\mathbf{Z}_{1}^{3}=-\frac{1}{2} t e^{-\sqrt{2} t} \frac{\partial}{\partial t}+\left(-\frac{3}{2} y e^{-\sqrt{2} t}+\frac{1}{\sqrt{2}} y t e^{-\sqrt{2} t}\right) \frac{\partial}{\partial y} .
$$

The nontrivial approximate Mei symmetry of Equation (2) has the form

$$
\mathbf{Z}^{3}=\mathbf{Z}_{0}^{3}+\epsilon \mathbf{Z}_{1}^{3}=\left(e^{-\sqrt{2} t} \frac{\partial}{\partial t}-y \sqrt{2} e^{-\sqrt{2} t} \frac{\partial}{\partial y}\right)+\epsilon\left(-\frac{1}{2} t e^{-\sqrt{2} t} \frac{\partial}{\partial t}+\left(-\frac{3}{2} y e^{-\sqrt{2} t}+\frac{1}{\sqrt{2}} y t e^{-\sqrt{2} t}\right) \frac{\partial}{\partial y}\right) .
$$

In a similar way, the remaining approximate Mei symmetries are obtained as

$$
\begin{aligned}
& \mathbf{Z}^{1}=\mathbf{Z}_{0}^{1}+\epsilon \mathbf{Z}_{1}^{1}=\frac{\partial}{\partial t}-\epsilon y \frac{\partial}{\partial y^{\prime}} \\
& \mathbf{Z}^{2}=\mathbf{Z}_{0}^{2}+\epsilon \mathbf{Z}_{1}^{2}=\left(e^{\sqrt{2} t} \frac{\partial}{\partial t}+y \sqrt{2} e^{\sqrt{2} t} \frac{\partial}{\partial y}\right)+\epsilon\left(-\frac{1}{2} t e^{\sqrt{2} t} \frac{\partial}{\partial t}+\left(-\frac{3}{2} y e^{\sqrt{2} t}-\frac{1}{\sqrt{2}} y t e^{\sqrt{2} t}\right) \frac{\partial}{\partial y}\right) \\
& \mathbf{Z}^{4}=\mathbf{Z}_{0}^{4}+\epsilon \mathbf{Z}_{1}^{4}=e^{-t} \frac{\partial}{\partial y}-\epsilon t e^{-t} \frac{\partial}{\partial y^{\prime}} \\
& \mathbf{Z}^{5}=\mathbf{Z}_{0}^{5}+\epsilon \mathbf{Z}_{1}^{5}=e^{t} \frac{\partial}{\partial y}-\epsilon t e^{t} \frac{\partial}{\partial y}{ }^{\prime} \\
& \mathbf{Z}^{6}=\mathbf{Z}_{0}^{6}+\epsilon \mathbf{Z}_{1}^{6}=y \frac{\partial}{\partial y}-\epsilon 4 t y \frac{\partial}{\partial y} .
\end{aligned}
$$

\section{Mei Invariants}

Using Equations (7) and (8), the approximate first integrals, $I=I_{0}+\epsilon I_{1}$, are obtained as

$$
\begin{aligned}
& I^{1}=I_{0}^{1}+\epsilon I_{1}^{1}=0, \\
& I^{2}=I_{0}^{2}+\epsilon I_{1}^{2}=3 \sqrt{2} y^{2} e^{2 \sqrt{2} t}+\epsilon\left(-\frac{15}{2} y^{2} e^{2 \sqrt{2} t}+3 \sqrt{2} y^{2} t e^{2 \sqrt{2} t}\right) \\
& I^{3}=I_{0}^{3}+\epsilon I_{1}^{3}=-3 \sqrt{2} y^{2} e^{2-\sqrt{2} t}+\epsilon\left(-\frac{15}{2} y^{2} e^{-2 \sqrt{2} t}-3 \sqrt{2} y^{2} t e^{-2 \sqrt{2} t}\right), \\
& I^{4}=I_{0}^{4}+\epsilon I_{1}^{4}=-e^{-2 t}-\epsilon e^{-2 t} \\
& I^{5}=I_{0}^{5}+\epsilon I_{1}^{5}=e^{2 t}-\epsilon e^{2 t} \\
& I^{6}=I_{0}^{6}+\epsilon I_{1}^{6}=2 y y^{\prime}-\epsilon\left(-12 t y y^{\prime}-4 y^{2}\right) .
\end{aligned}
$$

\section{Conclusions and Discussions}

In this paper, approximate Mei symmetries and first integrals corresponding to the approximate Hamiltonian are studied. Formulae of obtaining these symmetries and invariants/first integrals for the approximate Hamiltonian are given in Theorems 1 and 2, respectively. The given example of the damped harmonic oscillator demonstrates the procedure developed in detail. A comparison of approximate Noether and approximate Mei symmetries for DHO is given in Table 1 that shows: 
- The number of approximate Mei symmetries is more than the number of approximate Noether symmetries;

- $\quad$ The Mei symmetry $\mathbf{Z}^{1}$ is also contained in the set of Noether symmetries;

- $\quad$ The other Mei symmetries, $\mathbf{Z}^{2}, \mathbf{Z}^{3}, \mathbf{Z}^{4}, \mathbf{Z}^{5}$, and $\mathbf{Z}^{6}$ are different from the Noether symmetries; therefore, there are new corresponding conserved quantities.

Table 1. Comparison between approximate Noether symmetries and approximate Mei symmetries for DHO.

\begin{tabular}{|c|c|}
\hline Approximate Noether Symmetries & Approximate Mei Symmetries \\
\hline $\begin{array}{c}\mathbf{X}^{1}=\frac{\partial}{\partial t}-\epsilon y \frac{\partial}{\partial y} \\
\mathbf{X}^{2}=\left(\cos 2 t \frac{\partial}{\partial t}-y \sin 2 t \frac{\partial}{\partial y}\right)+\epsilon\left(-y \cos 2 t \frac{\partial}{\partial y}\right) \\
\mathbf{X}^{3}=\left(\sin 2 t \frac{\partial}{\partial t}+y \cos 2 t \frac{\partial}{\partial y}\right)+\epsilon\left(-y \sin 2 t \frac{\partial}{\partial y}\right) \\
\mathbf{X}^{4}=\sin t \frac{\partial}{\partial y}-\epsilon t \sin t \frac{\partial}{\partial y} \\
\mathbf{X}^{5}=\cos t \frac{\partial}{\partial y}-\epsilon t \cos t \frac{\partial}{\partial y}\end{array}$ & $\begin{aligned} & \mathbf{Z}^{1}=\frac{\partial}{\partial t}-\epsilon y \frac{\partial}{\partial y} \\
& \mathbf{Z}^{2}=\left(e^{\sqrt{2} t} \frac{\partial}{\partial t}+y \sqrt{2} e^{\sqrt{2} t} \frac{\partial}{\partial y}\right)+\epsilon\left(-\frac{1}{2} t e^{\sqrt{2} t} \frac{\partial}{\partial t}\right)+\left(-\frac{3}{2} y e^{\sqrt{2} t}-\frac{1}{\sqrt{2}} y t e^{\sqrt{2} t}\right) \frac{\partial}{\partial y} \\
& \mathbf{Z}^{3}=\left(e^{-\sqrt{2} t} \frac{\partial}{\partial t}+y \sqrt{2} e^{-\sqrt{2} t} \frac{\partial}{\partial y}\right)+\epsilon\left(-\frac{1}{2} t e^{-\sqrt{2} t} \frac{\partial}{\partial t}\right)+\left(-\frac{3}{2} y e^{-\sqrt{2} t}+\frac{1}{\sqrt{2}} y t e^{-\sqrt{2} t}\right) \frac{\partial}{\partial y} \\
& \mathbf{Z}^{4}=e^{-t} \frac{\partial}{\partial y}-\epsilon t e^{-t} \frac{\partial}{\partial y} \\
& \mathbf{Z}^{5}=e^{t} \frac{\partial}{\partial y}-\epsilon t e^{t} \frac{\partial}{\partial y} \\
& \mathbf{Z}^{6}=y \frac{\partial}{\partial y}-\epsilon 4 t y \frac{\partial}{\partial y}\end{aligned}$ \\
\hline
\end{tabular}

Author Contributions: U.K. and T.F. made equal contributions. All authors have read and agreed to the published version of the manuscript.

Funding: This research received no external funding.

Institutional Review Board Statement: Not applicable.

Informed Consent Statement: Not applicable.

Data Availability Statement: Not applicable.

Conflicts of Interest: The authors declare no conflict of interest.
Abbreviations
ODEs Ordinary differential equations
PDEs Partial differential equations
DHO Damped harmonic oscillator

The following abbreviations are used in this manuscript:

\section{References}

1. Bă̌kov, V.A.; Gazizov, R.K.; Ibragimov, N.H. Approximate symmetries. Math. USSR-Sb. 1989, 64, 427-441. [CrossRef]

2. Gazizov, R.K. Lie algebras of approximate symmetries. J. Nonlinear Math. Phys. 1996, 3, 96-101. [CrossRef]

3. Feroze, T.; Kara, A.H. Group theoretic methods for approximate invariants and Lagrangians for some classes of $y^{\prime \prime}+\epsilon F(t) y^{\prime}+y=$ $f\left(y, y^{\prime}\right)$. Int. J. Non-Linear Mech. 2002, 37, 275-280. [CrossRef]

4. Johnpillai, A.G.; Kara, A.H. Variational formulation of approximate symmetries and conservation laws. Int. J. Theor. Phys. 2001, 40, 1501-1509. [CrossRef]

5. Kara, A.H.; Mahomed, F.M.; Qadir, A. Approximate symmetries and conservation laws of the geodesic equations for the Schwarzschild metric. Nonlinear Dyn. 2008, 51, 183-188. [CrossRef]

6. Bai, Y.S.; Zhang, Q. Approximate symmetry analysis and approximate conservation laws of perturbed KdV equation. Adv. Math. Phys. 2018, 2018, 4743567. [CrossRef]

7. Camci, U. Approximate Noether gauge symmetries of the Bardeen model. Eur. Phys. J. 2014, 74, 1-8. [CrossRef]

8. Mei, F.-X. Form invariance of Lagrange system. J. Beijing Inst. Technol. 2000, 9, 120-124.

9. Mei, F.-X. Form invariance of Appell equations. Chin. Phys. 2001, 10, 177.

10. Wang, S.-Y.; Mei, F.-X. Form invariance and Lie symmetry of equations of non-holonomic systems. Chin. Phys. 2002, 11, 5.

11. Fang, J.-H. Mei symmetry and Lie symmetry of the rotational relativistic variable mass system. Commun. Theor. Phys. 2003, $40,269$.

12. Zhai, X.H.; Zhang, Y. Mei symmetry and new conserved quantities of time-Scale Birkhoff's equations. Complexity $2020,2020$. [CrossRef] 
13. Zhai, X.H.; Zhang, Y. Mei symmetry of time-scales Euler-Lagrange equations and its relation to Noether symmetry. Acta Phys. Pol. A 2019, 136, 439-443. [CrossRef]

14. Jia, L.; Wang, X.; Zhang, M.; Han, Y. Special Mei symmetry and approximate conserved quantity of Appell equations for a weakly nonholonomic system. Nonlinear Dyn. 2012, 69, 1807-1812. [CrossRef]

15. Han, Y.; Wang, X.; Zhang, M.; Jia, L. Lie symmetry and approximate Hojman conserved quantity of Appell equations for a weakly nonholonomic system. Nonlinear Dyn. 2013, 71, 401-408. [CrossRef]

16. Denman, H.H. Approximate invariants and Lagrangians for autonomous, weakly non-linear systems. Int. J. Non-Linear Mech. 1994, 29, 409-419. [CrossRef]

17. Fang, J.-H.; Yan, X.-H.; Li, H.; Chen, P.-S. Mei symmetry and Lie symmetry of relativistic Hamiltonian system. Commun. Theor. Phys. 2004, 42, 19. 